\title{
Clinical Impact of Cachexia in Head and Neck Cancer Patients Who Received Chemoradiotherapy
}

\author{
Naomi Hayashi' \\ Yasuyoshi Sato (D) \\ Yu Fujiwara (iD ${ }^{1,2}$ \\ Naoki Fukuda (D) \\ Xiaofei Wang (1D) \\ Kenji Nakano' \\ Testuya Urasaki' \\ Akihiro Ohmoto' \\ Makiko Ono' \\ Junichi Tomomatsu (D) \\ Yukiko Sato (iD) ${ }^{3}$ \\ Hiroki Mitani ${ }^{4}$ \\ Takashi Toshiyasu ${ }^{5}$ \\ Shunji Takahashi ${ }^{1}$ \\ 'Department of Medical Oncology, The \\ Cancer Institute Hospital of Japanese \\ Foundation for Cancer Research, Tokyo, \\ Japan; ${ }^{2}$ Department of Medicine, Icahn \\ School of Medicine at Mount Sinai, Mount \\ Sinai Beth Israel, New York City, NY, \\ USA; ${ }^{3}$ Pathology, The Cancer Institute \\ Hospital of Japanese Foundation for \\ Cancer Research, Tokyo, Japan; ${ }^{4}$ Head \\ and Neck Oncology, The Cancer Institute \\ Hospital of Japanese Foundation for \\ Cancer Research, Tokyo, Japan; \\ ${ }^{5}$ Radiation Oncology, The Cancer \\ Institute Hospital of Japanese Foundation \\ for Cancer Research, Tokyo, Japan
}

Correspondence: Yasuyoshi Sato The Cancer Institute Hospital of Japanese Foundation for Cancer Research, 3-8-3I Ariake, Koto, Tokyo, I35-8550, Japan

Tel +8I35200 I I I

Fax $+8|33520014|$

Email yasuyoshi.sato@jfcr.or.jp
Purpose: There have been few reports on the evaluation of cancer cachexia based on skeletal muscle mass index (SMI) in patients with head and neck cancer.

Patients and Methods: One hundred and ninety-two head and neck cancer patients were enrolled. In definitive and adjuvant chemoradiotherapy settings, clinical outcomes were compared between cachexia and non-cachexia patients.

Results: Forty patients were diagnosed with cachexia (20.8\%). In the definitive setting, overall survival (OS) was significantly shorter in the cachexia group (3-year OS: $50.0 \%$ vs $88.5 \% ; \mathrm{p}<0.01$ ), and multivariate analysis identified UICC stage IV, baseline albumin of $<4$ and cachexia as poor prognostic factors. However, cachexia was not significant in the adjuvant setting.

Conclusion: Cancer cachexia was negatively associated with prognosis in patients with HNC who received definitive chemoradiotherapy. Nutritional intervention during chemoradiotherapy may improve survival in these patients.

Keywords: head and neck cancer, squamous cell carcinoma of head and neck, sarcopenia, cachexia, muscle, skeletal, chemoradiotherapy, prognosis

\section{Introduction}

Head and neck cancer (HNC) was the seventh most common cancer worldwide in 2018 , accounting for $3 \%$ of all cancer types. ${ }^{1}$ Although risk factors for HNC are not completely understood, several factors such as smoking, alcohol, diet, and chronic viral infection, including human papillomavirus-associated oropharyngeal carcinoma, have been reported to increase its risk. ${ }^{2-4}$ Conversely, several factors, such as improvement in lifestyle, reduce the risk of cancer.

Sarcopenia, characterized as an age-related decline in muscle mass and strength, has been recognized to be an important prognostic factor in various types of cancers. $^{5-7}$ It has also been associated with severe toxicity in cancer patients with chemotherapy. ${ }^{8}$ Many studies about sarcopenia have been performed using computed tomography (CT) imaging. Sarcopenia based on CT imaging has been previously reported as an indicator of poor prognosis of HNC. ${ }^{9-11}$

Patients with cancer also have a risk of decline of muscle mass via cachexia. Cachexia is a multifactorial disease in which weight loss including skeletal muscle declines due to systemic inflammation from cancer. ${ }^{12}$ It is caused by the activation of cytokines such as tumor necrosis factor- $\alpha$ (TNF- $\alpha$ ), interleukin-6 (IL-6), and interferon- $\gamma$ (IF- $\gamma),{ }^{13}$ leading to a decline in protein synthesis and increase in proteolysis and lipolysis, frequently occurring in solid tumors, particularly gastric, 
pancreatic, lung cancer, and HNC. ${ }^{14}$ There have been many reports of associations between cachexia and prognosis on cancer patients; however, few studies strictly evaluated using CT imaging, opposed to sarcopenia. Actually, the studies about cachexia in HNC patients have been performed only based on nutritional status. ${ }^{15}$

Cachexia is often present in patients with head and neck squamous cell carcinoma (HNSCC) ${ }^{9}$ and progresses during concurrent chemoradiotherapy (CCRT), which is administered for a few months. There are two settings of CCRT, definitive and adjuvant. Definitive CCRT is performed with locally advanced HNSCC to achieve cure by CCRT alone; Patients sometimes select definitive CCRT for laryngeal preservation and avoiding change in appearance or other quality of life degradation even if their lesions are resectable. Adjuvant CCRT is performed with post-operative high-risk HNSCC to reduce recurrence risk. Although it is important to assess their condition, little is known about clinical impact of cachexia in HNSCC patients who received CCRT. In this study, we evaluated the association between cachexia (including CT imaging evaluation) and prognosis in patients with HNSCC who received CCRT.

\section{Patients and Methods}

\section{Patients}

We retrospectively analyzed prospectively collected data from consecutive patients with HNSCC who were initiated with concurrent chemoradiotherapy (CCRT) with cisplatin at the Cancer Institute Hospital of Japanese Foundation for Cancer Research (JFCR) between January 2015 and December 2018. Patients' data included the following characteristics: age, sex, height, weight, smoking status, Eastern Cooperative Oncology Group performance status (PS, ECOG PS), location of the primary tumor, histological diagnosis (including p16 protein expression), clinical stage (based on UICC TNM classification), baseline albumin, total dose of cisplatin, radiation details, and pretreatment CT findings. These factors were categorized for analysis as follows: age: $<65$ years or $\geq 65$ years; sex: male or female; ECOG PS: 0 or $\geq 1$; smoking: current/former or never; primary tumor site: nasopharynx, oropharynx, or other; clinical stage: $1-3$ or 4 ; and baseline albumin: $<4$ or $\geq 4$. Cachexia was defined when 1) weight loss of $>5 \%$ in 6 months (in the absence of simple starvation), 2) body mass index (BMI) of $<20 \mathrm{~kg} / \mathrm{m}^{2}$ and weight loss of $>2 \%$, or 3) sarcopenia and weight loss of $>2 \%$. $^{12,16}$ We analyzed CT images, including the CT component of whole-body PET-CT scans, at the level of the third lumbar vertebra (L3). Skeletal muscle mass area was calculated using the Volume Analyzer SYNAPSE VINCENT image analysis system (Fujifilm Medical, Tokyo, Japan). ${ }^{17}$ Abdominal skeletal muscle includes the psoas major, paraspinals (erector spinae and quadratus lumborum), and muscles of the abdominal wall (transversus abdominus, external and internal obliques, and rectus abdominus). The skeletal muscle mass index (SMI) which normalizes skeletal muscle area adjusted by height was used as an indicator of sarcopenia. ${ }^{8}$ The cross-sectional area of skeletal muscle at L3 was measured using the SYNAPSE VINCENT with Hounsfield unit thresholds of -30 to +150 . After segmentation, minor manual measurements were performed as required. Sarcopenia was diagnosed with reference to Martin's cut-off value: SMI of $<43 \mathrm{~cm}^{2} / \mathrm{m}^{2}$ for men with BMI $<25 \mathrm{~kg} / \mathrm{m}^{2}$ or $<53 \mathrm{~cm}^{2} / \mathrm{m}^{2}$ for men with BMI $\geq 25 \mathrm{~kg} / \mathrm{m}^{2}$ and $<41 \mathrm{~cm}^{2} / \mathrm{m}^{2}$ for women. ${ }^{18}$

Cisplatin was administered at a dose of $80 \mathrm{mg} / \mathrm{m}^{2}$ (from 2012 to August 2015) or $100 \mathrm{mg} / \mathrm{m}^{2}$ (from August 2015 to 2018) every 3 weeks for a total of three cycles. Elderly patients and those with reduced organ function received a reduced cisplatin dose according to the discretion of their physician. When patients complicated with intolerable adverse events from cisplatin, skip, delay, or dose reduction of the second and/or third cisplatin cycle were permitted. Radiotherapy was performed as 3-dimensional conformal radiotherapy (3D-CRT) or intensity-modified radiotherapy (IMRT) with the conventional fraction, 2-2.12 Gy per fraction, once per day, five times per week. Prophylactic percutaneous endoscopic gastrostomy was performed unless particular reasons forbid it (such as refusal by the patient or past history of gastrectomy). Follow-up examinations using enhanced CT and measurements of blood biochemistry and serum tumor markers were performed approximately every 3 months after CCRT.

\section{Statistical Analysis}

Progression-free survival (PFS) and overall survival (OS) were estimated using the Kaplan-Meier method and Log rank test. Data were censored on January 31, 2021. Patients who were lost to follow-up were censored at the date of last contact or follow-up. PFS was calculated from the date of radiation initiation to the date of disease relapse, disease progression or death from any cause. OS was calculated from the date of radiation initiation to the date of death from any cause. Patients who were alive on January 31, 2021, were censored for OS analysis. We estimated survival 
curves by definitive or adjuvant CCRT using the KaplanMeier method and Log rank test. We performed univariate and multivariate analyses to estimate factors potentially prognostic for PFS and OS by calculating hazard ratios (HRs) using the Cox proportional hazards model. The level of significance was set at $p$ value of $<0.05$ for univariate and multivariate analysis, which was two-sided. All statistical analyses were performed using EZR (Saitama Medical Center, Jichi Medical University, Saitama, Japan), which is a modified interface for R software (www.r-project.org). ${ }^{19}$

\section{Results}

\section{Patients' Characteristics}

Three hundred and forty-four patients were included. Of all the patients, 152 were excluded because presence or absence of cachexia could not be evaluated based on criteria. Overall, 192-patient cohort comprised 40 (20.8\%) with cachexia patients and 152 (79.2\%) without cachexia patients with a median age of 61 years (range, 20-78 years). The median observation time was 38.6 months (range, 4.6-68.9 months) after the initiation of CCRT. Among these, 39 patients could not complete CCRT because of toxicity. The median radiation dose was 66 Gy and the median cisplatin dose was $280 \mathrm{mg}$, which differed between 2015 and 20162018 ( $240 \mathrm{mg}$ and $300 \mathrm{mg}$, respectively) owing to alteration of the standard dose. At the time of censoring, 43 patients (22.4\%) had died from the primary disease. The-192 patients were divided into definitive CCRT $(\mathrm{n}=148)$ and adjuvant CCRT $(\mathrm{n}=44)$ groups. For data analysis, the two groups were categorized into the following four: 1) definitive CCRT with cachexia, 2) definitive CCRT without cachexia, 3) adjuvant CCRT with cachexia, and 4) adjuvant CCRT without cachexia (Figure 1). The characteristics of the 192 patients classified into four groups are shown in Table 1. Sixty-six patients were oropharyngeal cancer patients. Of 66 patients, 42 patients $(73.6 \%)$ in definitive CCRT and 2 patients (40\%) in adjuvant CCRT were P16 positive. The median interval between $\mathrm{CT}$ imaging and initial chemotherapy was 28 days (range: 1-60). In the definitive CCRT setting, the groups with cachexia and without cachexia significantly differed in PS and baseline albumin. In the adjuvant CCRT setting, the two groups showed no difference.

\section{Long-Term Outcomes}

Figure 2 shows PFS and OS stratified by cachexia and CCRT setting. There were significant differences for definitive CCRT between cachexia and non-cachexia groups: PFS (15.4 months vs NA; HR, 4.01; 95\% CI, 2.15-7.47; $\mathrm{p}<0.01)$ and OS (42.8 months vs NA; HR, 5.81; 95\% CI, 2.72-12.41; $\mathrm{p}<0.01)$. Three-year survival rate and local recurrence rate were significantly different in the cachexia group $(50.0 \%$ vs $88.5 \%$; $<0.01$ and $26.9 \%$ vs $6.6 \%, \mathrm{p}<0.01)$. In the adjuvant CCRT setting, there were no significant differences between the two groups for PFS (NA vs 19.0 months; HR, 0.48; 95\% CI, 0.18$1.31 ; \mathrm{p}=0.15$ ) or OS (NA vs NA; HR, $0.64 ; 95 \% \mathrm{CI}$, $0.20-2.01 ; \mathrm{p}=0.45)$. Three-year survival rate and local

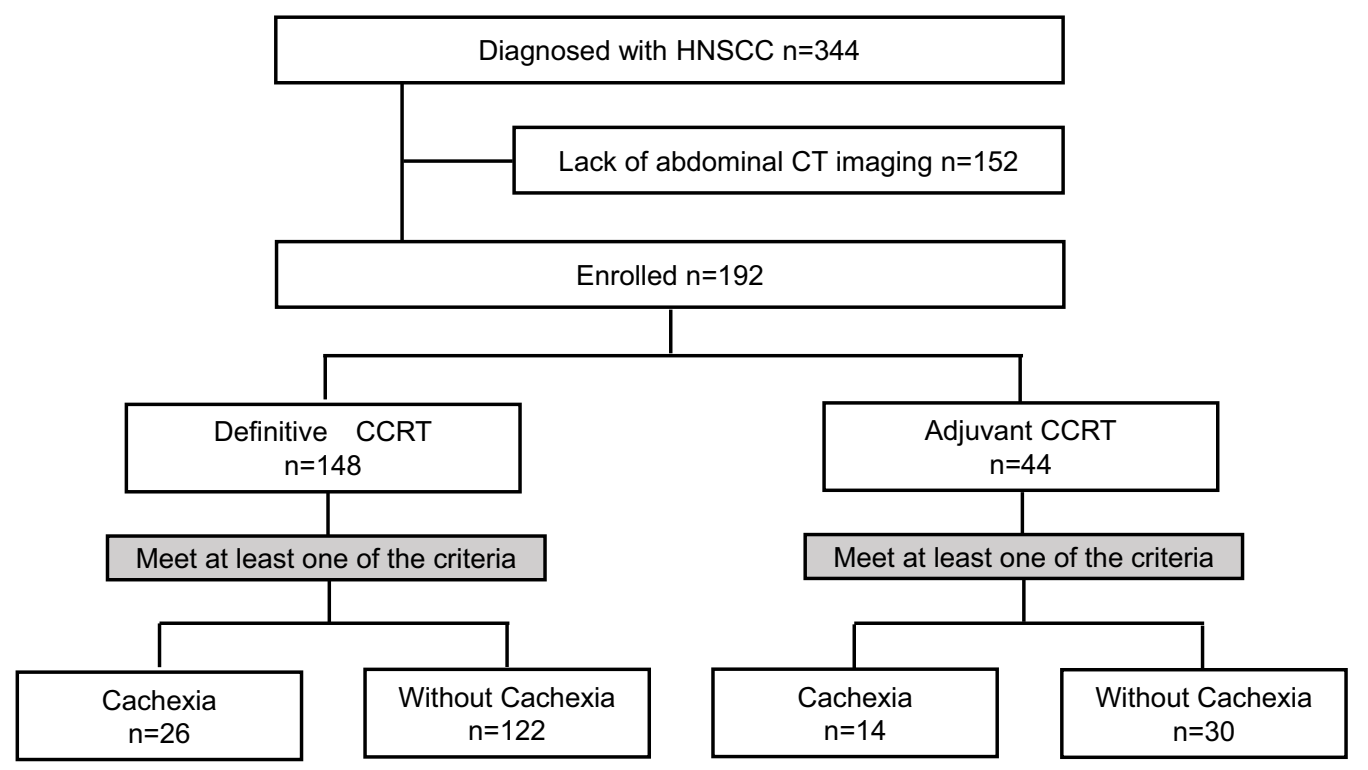

Figure I CONSORT diagram of this study. 
Table I Patients' Characteristics

\begin{tabular}{|c|c|c|c|c|c|c|c|c|c|c|c|}
\hline & & \multicolumn{5}{|c|}{ Definitive CCRT } & \multicolumn{5}{|c|}{ Adjuvant CCRT } \\
\hline & & \multicolumn{2}{|c|}{$\begin{array}{l}\text { Cachexia } \\
n=26\end{array}$} & \multicolumn{2}{|c|}{$\begin{array}{l}\text { Without Cachexia } \\
n n=122\end{array}$} & \multirow[t]{2}{*}{ P value } & \multicolumn{2}{|c|}{$\begin{array}{c}\text { Cachexia } \\
n=14\end{array}$} & \multicolumn{2}{|c|}{$\begin{array}{c}\text { Without } \\
\text { Cachexia } n=30\end{array}$} & \multirow[t]{2}{*}{$P$ value } \\
\hline & & $\mathbf{n}$ & (\%) & $\mathbf{n}$ & (\%) & & $\mathbf{n}$ & (\%) & $\mathbf{n}$ & (\%) & \\
\hline Age & Median (range) & \multicolumn{2}{|c|}{$58.5(37-73)$} & \multicolumn{2}{|c|}{$61.0(36-78)$} & 0.29 & \multicolumn{2}{|c|}{$61.0(43-72)$} & \multicolumn{2}{|c|}{$60.0(20-76)$} & 0.63 \\
\hline \multirow[t]{2}{*}{ Sex } & Male & 21 & $(80.8)$ & 98 & $(80.3)$ & 1.00 & 11 & $(78.6)$ & 29 & $(96.7)$ & 0.08 \\
\hline & Female & 5 & $(19.2)$ & 24 & $(19.7)$ & & 3 & $(21.4)$ & 1 & $(3.3)$ & \\
\hline \multirow[t]{2}{*}{ PS } & 0 & 18 & $(69.2)$ & 112 & $(91.8)$ & $<0.01$ & 10 & $(7 I .4)$ & 24 & $(80.0)$ & 0.70 \\
\hline & $\geq 1$ & 8 & $(30.8)$ & 10 & $(8.2)$ & & 4 & $(28.6)$ & 6 & $(20.0)$ & \\
\hline \multirow[t]{2}{*}{ Smoking } & Yes & 21 & $(80.8)$ & 98 & $(80.3)$ & 1.00 & 10 & (7I.4) & 24 & $(80.0)$ & 0.70 \\
\hline & No & 5 & $(19.2)$ & 24 & $(19.7)$ & & 4 & $(28.6)$ & 6 & $(20.0)$ & \\
\hline \multirow[t]{2}{*}{ Primary site } & Naso/oropharynx & 10 & $(38.5)$ & 62 & $(50.8)$ & 0.28 & 2 & $(14.3)$ & 4 & $(13.3)$ & 1.00 \\
\hline & Other & 16 & $(6 I .5)$ & 60 & $(49.2)$ & & 12 & $(85.7)$ & 26 & $(86.7)$ & \\
\hline \multirow[t]{3}{*}{ PI6 } & Positive & 6 & $(23.1)$ & 44 & $(36.1)$ & 0.45 & I & $(7.1)$ & 5 & $(16.7)$ & 0.63 \\
\hline & Negative & 14 & $(53.8)$ & 61 & $(50.0)$ & & 9 & $(64.3)$ & 15 & $(50.0)$ & \\
\hline & Unknown & 6 & $(23.1)$ & 17 & $(13.0)$ & & 4 & $(28.6)$ & 6 & $(20.0)$ & \\
\hline \multirow[t]{2}{*}{ Stage } & I-III & 12 & $(46.2)$ & 89 & $(73.0)$ & 0.01 & 4 & $(28.6)$ & 10 & $(33.3)$ & 1.00 \\
\hline & IV & 14 & $(53.8)$ & 33 & $(27.0)$ & & 10 & $(7 I .4)$ & 20 & $(66.6)$ & \\
\hline \multirow[t]{3}{*}{ Sarcopenia } & Yes & 12 & $(46.2)$ & 33 & $(27.0)$ & $<0.01$ & 2 & $(14.3)$ & 6 & $(20.0)$ & 0.56 \\
\hline & No & 7 & $(26.9)$ & 87 & (7I.3) & & 3 & $(21.4)$ & 24 & $(80.0)$ & \\
\hline & Unknown & 7 & $(26.9)$ & 2 & $(1.6)$ & & 9 & $(64.3)$ & 0 & $(0.0)$ & \\
\hline Baseline albumin & Median (range) & \multicolumn{2}{|c|}{$4.1(2.5-4.3)$} & \multicolumn{2}{|c|}{$4.2(3.3-4.9)$} & $<0.01$ & \multicolumn{2}{|c|}{$4.0(3.5-4.6)$} & \multicolumn{2}{|c|}{$4.1(2.1-4.5)$} & 0.89 \\
\hline CCRT dose & Median & \multicolumn{2}{|c|}{240} & \multicolumn{2}{|c|}{300} & 0.22 & \multicolumn{2}{|c|}{240} & \multicolumn{2}{|c|}{260} & 0.42 \\
\hline RT dose & Median (range) & \multicolumn{2}{|c|}{$66(60-66)$} & \multicolumn{2}{|c|}{$66(60-70)$} & 0.52 & \multicolumn{2}{|c|}{$66(60-70)$} & \multicolumn{2}{|c|}{$66(46-70)$} & 0.16 \\
\hline
\end{tabular}

Abbreviations: PS, performance status; CCRT, concurrent chemoradiotherapy; RT, radiation therapy.

recurrence rate were not significantly different between the two groups $(71.4 \%$ vs $60.0 \%, p=0.52$ and $14.3 \%$ vs $30.0 \%, \mathrm{p}=0.45)$.

\section{Risk Factor of Prognosis}

In the definitive CCRT setting, multivariate Cox proportional hazard analysis indicated that the stage (HR, 2.74; 95\% CI, 1.46-5.12; $<<0.01)$, baseline albumin (HR, 2.83; 95\% CI, 1.53-5.23; p < 0.01), and cachexia (HR, 3.51; 95\% CI, 1.65-6.01; $\mathrm{p}<0.01)$ were independent risk factors for PFS. Independent risk factors for poor OS were stage (HR, 2.64; 95\% CI, 1.15-6.07; $\mathrm{p}=0.02$ ), baseline albumin (HR, 3.91; 95\% CI, 1.78-8.59; p < 0.01), and cachexia (HR, 4.31; 95\% CI, 1.93-9.61; p < 0.01). Analysis by adjuvant CCRT setting revealed no independent predictive factors (Tables 2 and 3 ).

\section{Adverse Events}

The frequency of grade $3-4$ adverse events is presented in Table 4. In definitive CCRT, all adverse events were strongly associated with cachexia. Among the grade 3-4 adverse events, the frequency of anemia, leukopenia and neutropenia had significant difference between the two groups $(23.8 \%$ and $0.8 \%, \mathrm{p}<0.01,53.8 \%$ and $32.7 \%$, $\mathrm{p}<0.01,23.1 \%$ and $19.7 \%, \mathrm{p}=0.03$, respectively). In the adjuvant CCRT setting, there were no significant differences in the frequency of severe adverse events.

\section{Discussion}

We investigated the association between cancer cachexia and prognosis in patients with HNSCC who received chemoradiotherapy. The results demonstrated that cachexia was an independent predictor of poor prognosis, particularly in the 

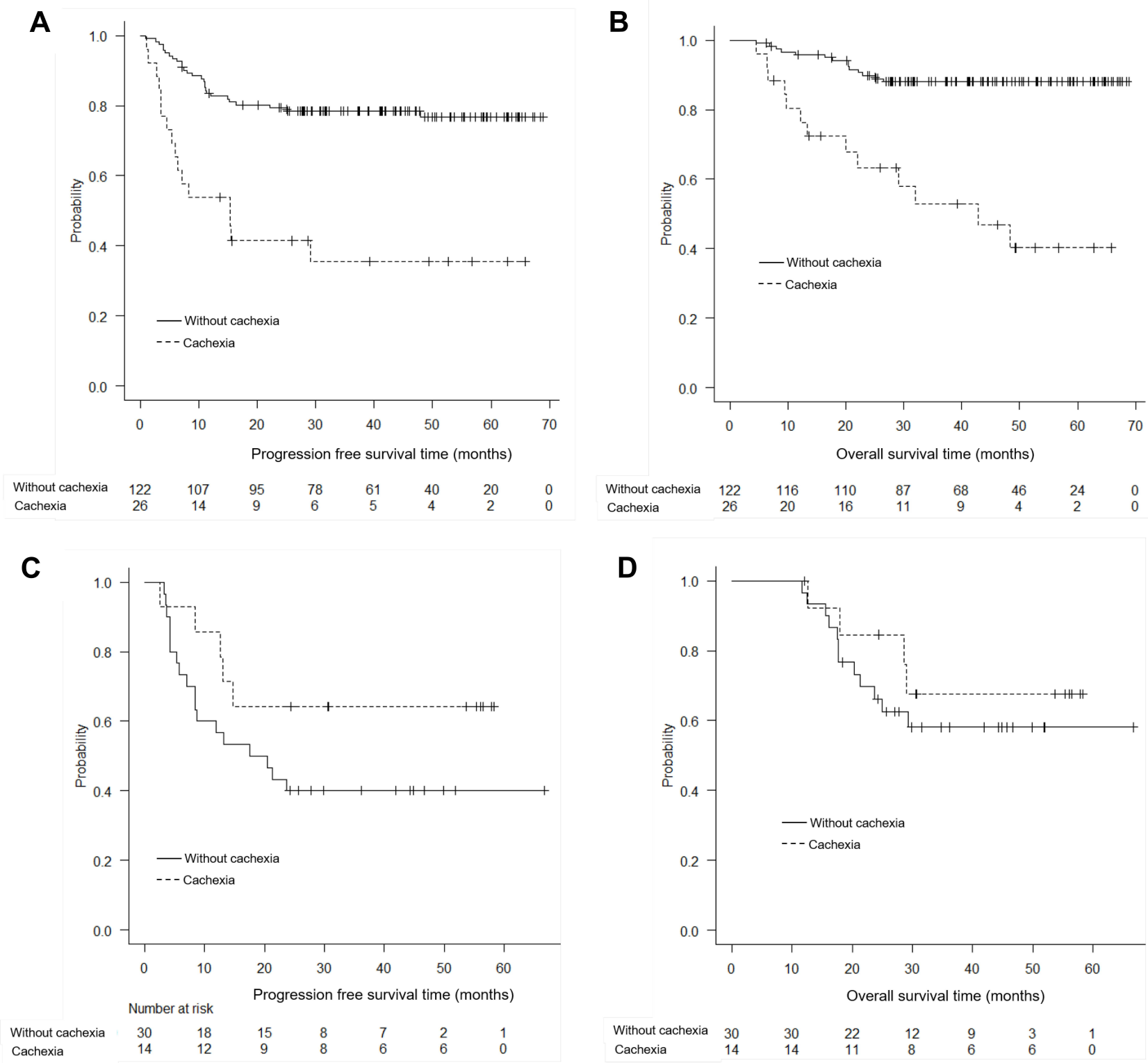

Figure 2 Kaplan-Meier curves for progression-free survival and overall survival according to treatment setting. Definitive CCRT-progression-free survival (PFS) (A), definitive CCRT-overall survival (OS) (B), adjuvant CCRT-progression-PFS (C) and adjuvant CCRT-OS (D) comparing patients with cachexia vs those without cachexia.

definitive setting. Grade 3-4 adverse events occurred more frequently in patients with cachexia.

To the best of our knowledge, this is the first study with a large sample size to evaluate the influence of cachexia in patients with HNSCC who received CCRT, including CT imaging at the level of L3. Our findings showed that cachexia is an important prognostic factor regardless of stage. A previous retrospective study could not show a significant difference between sarcopenia and nonsarcopenia in prognosis among HNSCC patients receiving CCRT. ${ }^{9}$ In the present study, of the patients who received definitive CCRT, 139 patients evaluated both cachexia and sarcopenia were also analyzed. The hazard ratio of OS was 2.24 in sarcopenia $(\mathrm{p}=0.053)$ and 4.9 in cachexia $(\mathrm{p}<0.01)$ (Supplemental Figure 1). These results suggested that evaluation of cachexia could be a better predictor for prognosis than that of sarcopenia in HNSCC patients before CCRT. It is biologically plausible that background systemic inflammation or weight loss from causes other than skeletal muscle in cachexia would have led to this result.

Previous reports showed, in sarcopenia, owing to reduced age-related changes in body composition, polar drugs that are mainly water-soluble tend to have smaller volumes of distribution, resulting in higher serum levels in 
Table 2 Univariate and Multivariate Analysis for PFS

\begin{tabular}{|c|c|c|c|c|c|c|c|c|c|}
\hline & \multicolumn{6}{|c|}{ Definitive CCRT } & \multirow{2}{*}{\multicolumn{3}{|c|}{$\frac{\text { Adjuvant CCRT }}{\text { Univariate }}$}} \\
\hline & \multicolumn{3}{|c|}{ Univariate } & \multicolumn{3}{|c|}{ Multivariate } & & & \\
\hline & HR & $95 \% \mathrm{Cl}$ & $P$ value & HR & $95 \% \mathrm{Cl}$ & $P$ value & HR & $95 \% \mathrm{Cl}$ & $P$ value \\
\hline Age, $\geq 65$ & 0.95 & $0.50-1.80$ & 0.88 & 1.01 & $0.52-1.95$ & 0.96 & 0.46 & $0.18-1.18$ & 0.10 \\
\hline Sex, Male & 1.36 & $0.60-3.07$ & 0.44 & 1.54 & $0.67-3.51$ & 0.30 & 1.08 & $0.25-4.60$ & 0.91 \\
\hline PS, $\geq 1$ & 1.94 & $0.89-4.19$ & 0.09 & & & & 1.66 & $0.68-4.07$ & 0.26 \\
\hline Smoking, Current/former & 0.91 & $0.44-1.91$ & 0.83 & & & & 0.66 & $0.26-1.68$ & 0.38 \\
\hline Primarysite, Naso/oropharynx & 0.55 & $0.29-1.03$ & 0.06 & & & & 0.96 & $0.28-3.24$ & 0.95 \\
\hline Stage, IV & 3.40 & $1.86-6.22$ & $<0.01$ & 2.74 & $1.46-5.12$ & $<0.01$ & 2.83 & $0.96-8.35$ & 0.05 \\
\hline Baseline albumin, $<4$ & 2.97 & $1.62-5.44$ & $<0.01$ & 2.83 & $1.53-5.23$ & $<0.01$ & 0.70 & $0.30-1.60$ & 0.40 \\
\hline Cachexia & 4.01 & $2.15-7.47$ & $<0.01$ & 3.51 & $1.65-6.01$ & $<0.01$ & 0.48 & $0.18-1.31$ & 0.15 \\
\hline
\end{tabular}

Abbreviations: PS, performance status; CCRT, concurrent chemoradiotherapy.

Table 3 Univariate and Multivariate Analyses for OS

\begin{tabular}{|c|c|c|c|c|c|c|c|c|c|}
\hline & \multicolumn{6}{|c|}{ Definitive CCRT } & \multirow{2}{*}{\multicolumn{3}{|c|}{$\begin{array}{c}\text { Adjuvant CCRT } \\
\text { Univariate }\end{array}$}} \\
\hline & \multicolumn{3}{|c|}{ Univariate } & \multicolumn{3}{|c|}{ Multivariate } & & & \\
\hline & HR & $95 \% \mathrm{Cl}$ & $P$ value & HR & $95 \% \mathrm{Cl}$ & $P$ value & HR & $95 \% \mathrm{Cl}$ & $P$ value \\
\hline Age, $\geq 65$ & 0.85 & $0.37-1.94$ & 0.69 & 0.89 & $0.38-2.11$ & 0.80 & 0.26 & $0.07-0.95$ & 0.04 \\
\hline Sex, Male & 1.56 & $0.54-4.52$ & 0.41 & 2.10 & $0.68-6.41$ & 0.19 & 1.45 & $0.19-11.0$ & 0.71 \\
\hline PS, $\geq$ I & 2.56 & $1.04-6.40$ & 0.04 & 1.23 & $0.47-3.20$ & 0.66 & 0.68 & $0.19-2.39$ & 0.54 \\
\hline Smoking, Current/former & 0.67 & $0.28-1.60$ & 0.37 & & & & 1.03 & $0.29-3.62$ & 0.96 \\
\hline Primarysite, Naso/oropharynx & 0.58 & $0.26-01.28$ & 0.18 & & & & 0.82 & $0.18-3.01$ & 0.79 \\
\hline Stage, IV & 3.99 & I.83-8.73 & $<0.01$ & 2.64 & I.15-6.07 & 0.02 & 3.77 & $0.85-16.6$ & 0.07 \\
\hline Baseline albumin, $<4$ & 4.01 & $1.88-8.56$ & $<0.01$ & 3.91 & I.78-8.59 & $<0.01$ & 0.62 & $0.22-1.72$ & 0.36 \\
\hline Cachexia & 5.81 & $2.72-12.41$ & $<0.01$ & 4.31 & $\mid .93-9.61$ & $<0.01$ & 0.64 & $0.20-2.01$ & 0.45 \\
\hline
\end{tabular}

Abbreviations: PS, performance status; CCRT, concurrent chemoradiotherapy.

Table 4 Frequency of Adverse Events of More Than Grade 3

\begin{tabular}{|c|c|c|c|c|c|c|c|c|c|c|}
\hline & \multicolumn{5}{|c|}{ Definitive CCRT } & \multicolumn{5}{|c|}{ Adjuvant CCRT } \\
\hline & \multicolumn{2}{|c|}{ Cachexia $n=26$} & \multicolumn{2}{|c|}{ Without Cachexia $n=\mid 22$} & \multirow[t]{2}{*}{$P$ value } & \multicolumn{2}{|c|}{ Cachexia $n=14$} & \multicolumn{2}{|c|}{ Without Cachexia $n=30$} & \multirow[t]{2}{*}{$P$ value } \\
\hline & $\mathbf{n}$ & (\%) & $\mathbf{n}$ & (\%) & & $\mathbf{n}$ & (\%) & $\mathbf{n}$ & (\%) & \\
\hline Anemia & 6 & $(23.1)$ & $\mathrm{I}$ & $(0.8)$ & $<0.01$ & 0 & $(0.0)$ & 4 & $(13.3)$ & 0.20 \\
\hline Leukopenia & 14 & $(53.8)$ & 40 & $(32.7)$ & $<0.01$ & 8 & $(57.1)$ & 14 & $(46.7)$ & 0.89 \\
\hline Neutropenia & 6 & $(23.1)$ & 24 & $(19.7)$ & 0.03 & 6 & $(42.9)$ & 13 & $(43.3)$ & 0.95 \\
\hline Thrombocytopenia & 2 & (7.7) & I & $(0.8)$ & 0.09 & 0 & $(0.0)$ & 1 & (3.3) & 0.17 \\
\hline Liver failure & 0 & $(0.0)$ & I & $(0.8)$ & 0.38 & I & (7.1) & 0 & $(0.0)$ & 0.17 \\
\hline Renal failure & 0 & $(0.0)$ & 0 & $(0.0)$ & 1.00 & 0 & $(0.0)$ & 0 & $(0.0)$ & 1.00 \\
\hline
\end{tabular}

Abbreviation: CCRT, concurrent chemoradiotherapy.

sarcopenia; ${ }^{20}$ and poor prognosis in elderly patients with cachexia may result from dose reduction owing to anticancer drug therapy. ${ }^{21}$ In the same study, the frequency of grade 3-4 adverse events was high in cachexia. In our study, frequency of grade 3-4 some hepatotoxicity was observed highly in cachexia, especially in definitive CCRT. The frequency of other adverse events was low, to begin with, so it was considered that there was no 
difference. It has been reported that cisplatin may improve prognosis at a total dose of $\geq 200 \mathrm{mg}$. $^{22,23}$ Owing to dose reduction or poor PS, the median cisplatin total dose was $240 \mathrm{mg}$ in cachexia without renal failure. The change in standard dose for cisplatin made in 2015 did not affect the distribution of cachexia. Cachexia was a significantly poor prognostic factor, regardless of cisplatin dose.

Recently, in Japan, anamorelin - a high-affinity, selective agonist of the ghrelin receptor-was approved for the treatment of cachexia in patients with non-small-cell lung carcinoma, gastric cancer, pancreatic cancer, and colorectal cancer but not in those with HNC. ${ }^{24}$ Thus, nutritional intervention may be one of the most effective approaches for the early stage of cachexia in HNSCC. However, several reports that evaluated the effects of nutritional intervention have failed to show any improvement for survival in HNSCC as a setting of both definitive and adjuvant chemotherapy. ${ }^{25}$ Although nutritional status appears to improve with dietary counseling, megestrol acetate, and prophylactic enteral tube feeding, there is no evidence that nutritional intervention can improve the prognosis of patients with HNC. ${ }^{26-28}$ However, these studies include few patients treated with chemotherapy; the results are limited; thus, further study in this area is warranted. Our data, at least, support the potential of a nutrition-based approach.

In this study, unlike several previous reports, we separately analyzed definitive and adjuvant CCRT settings. As the standard treatment for HNSCC is surgery plus adjuvant CCRT (in cases at high risk for recurrence) or definitive CCRT, several studies included both groups in their analysis. Our study showed that cachexia was an independent prognostic factor in patients who received definitive CCRT. This may be because definitive CCRT has a wide range of radiation, including cervical lymph nodes, causing patients to experience loss of oral intake. Evaluation of cachexia in the adjuvant CCRT setting was difficult because its presence or absence was determined using preoperative abdominal $\mathrm{CT}$ imaging findings. Postoperative imaging would have been more accurate; however, it provides no clinical benefit.

Several limitations of this study should be acknowledged. First, this was a single-institution retrospective study with potential selection bias and a short follow-up time. Second, data on adverse events associated with nutrition such as oral mucositis, appetite loss, or nausea were not included in our database because only objectively assessable adverse events such as hematotoxicity were collected. Third, there was a lack of data on sarcopenia because abdominal CT imaging is not routinely performed. Sarcopenia is normally diagnosed based on the skeletal mass index at L3 using one of the two cut-off value methods (Prado or Martin); ${ }^{8,18}$ we used Martin's. These cut-off values derived from Western studies are unsuitable for Asian patients with BMI of $<25 \mathrm{~kg} / \mathrm{m}^{2}$. An Asian sarcopenia working group proposed using dual-energy $\mathrm{X}$-ray absorptiometry or bioimpedance measurements for the diagnosis of sarcopenia. ${ }^{29}$ However, these are not performed routinely in real-world clinical practice and do not fit in retrospective analyses. Nutritional indexes, such as Glasgow Prognostic Scale, are considered as candidate surrogate markers for cachexia syndrome. ${ }^{15}$ However, in actuality, no reports are verifying the association between nutritional index and cachexia. Therefore, we evaluated cachexia stick to the definition using $\mathrm{CT}$ imaging at the level of L3 for sarcopenia diagnosis in this study. In actuality, our data of criteria used for diagnosis cachexia in individual patients showed there was only one patient diagnosed cachexia by criteria of "sarcopenia and weight loss of $>2 \%$ " (Supplemental Table 1). That could support the validity of our study although there were some missing data. And we are planning further research on whether the nutritional index can be an indicator of cachexia. This may make it possible to evaluate cachexia in any country where CT image analyses were not accessible in the public health system.

\section{Conclusion}

We have retrospectively evaluated the association between cancer cachexia and prognosis in patients with HNSCC who received CCRT. Cachexia was an independent poor prognostic factor in patients with HNSCC who received definitive CCRT. Ongoing nutritional intervention before CCRT can help improve survival. Further study is warranted.

\section{Ethics Approval and Consent to Participate}

The requirement for informed consent was waived because data were reported anonymously. The study was approved by the Institutional Review Board of The Cancer Institute Hospital of JFCR (2021-1037). All procedures were performed in accordance with the ethical standards of the responsible committees on human experimentation 
(institutional and national) and with the Helsinki Declaration of 1964 and subsequent versions.

\section{Acknowledgments}

We thank to medical staff of the Department of Head and Neck Surgery, and Radiation Oncology, The Cancer Institute Hospital of Japanese Foundation for Cancer Research for their support during this study. We thank Edanz (https://jp.edanz.com/ac) for editing a draft of this manuscript.

\section{Funding}

This research received no specific grant from any funding agency in the public, commercial, or not-for-profit sectors.

\section{Disclosure}

$\mathrm{NH}$ reports personal fees from TAIHO Pharmaceutical Co., Ltd. YS reports personal fees from ONO Pharmaceutical Co., Ltd., Bristol-Myers Squibb Company, MSD K.K., and TAIHO Pharmaceutical Co., Ltd. NF reports personal fees from Eisai. JT reports personal fees from Eisai. ST reports grants and personal fees from ONO Pharmaceutical Co., Ltd., Bristol-Myers Squibb, MSD, AstraZeneca, Chugai, and BAYER. The authors report no other conflicts of interest in this work.

\section{References}

1. Bray F, Ferlay J, Soerjomataram I, et al. Global cancer statistics 2018: GLOBOCAN estimates of incidence and mortality worldwide for 36 cancers in 185 countries. CA Cancer J Clin. 2018;68(6):394-424. doi: $10.3322 /$ caac. 21492

2. Radoï L, Luce D. A review of risk factors for oral cavity cancer: the importance of a standardized case definition. Community Dent Oral Epidemiol. 2013;41(2):97-109. doi:10.1111/j.1600-0528.2012.00710.

$$
\mathrm{x}
$$

3. Turati F, Garavello W, Tramacere I, et al. A meta-analysis of alcohol drinking and oral and pharyngeal cancers: results from subgroup analyses. Alcohol Alcohol. 2013;48(1):107-118. doi:10.1093/alcalc/ ags 100

4. Chaturvedi AK, Anderson WF, Lortet-Tieulent J, et al. Worldwide trends in incidence rates for oral cavity and oropharyngeal cancers. $J$ Clin Oncol. 2013;31(36):4550-4559. doi:10.1200/JCO.20 13.50 .3870

5. Chindapasirt J. Mini-review sarcopenia in cancer patients. Asian Pac J Cancer Prev. 2015;16:8075-8077.

6. Reynolds P. Characteristics of ageing skeletal muscle. Physiother Theory Pract. 1991;7(3):157-162. doi:10.3109/09593989109106967

7. Larsson L. Histochemical characteristics of human skeletal muscle during aging. Acta Physiol Scand. 1983;117(3):469-471. doi:10.1111/j.1748-1716.1983.tb00024.x

8. Prado CM, Lieffers JR, McCargar LJ, et al. Prevalence and clinical implications of sarcopenic obesity in patients with solid tumours of the respiratory and gastrointestinal tracts: a population-based study. Lancet Oncol. 2008;9(7):629-635. doi:10.1016/S1470-2045(08)70153-0
9. Wendrich AW, Swartz JE, Bril SI, et al. Low skeletal muscle mass is a predictive factor for chemotherapy dose-limiting toxicity in patients with locally advanced head and neck cancer. Oral Oncol. 2017;71:26-33. doi:10.1016/j.oraloncology.2017.05.012

10. Ganju RG, Morse R, Hoover A, et al. The impact of sarcopenia on tolerance of radiation and outcome in patients with head and neck cancer receiving chemoradiation. Radiother Oncol. 2019;137 (2019):117-124. doi:10.1016/j.radonc.2019.04.023

11. Grossberg AJ, Chamchod S, Fuller CD, et al. Association of body composition with survival and locoregional control of radiotherapy-treated head and neck squamous cell carcinoma. JAMA Oncol. 2016;2(6):782-789. doi:10.1001/jamaoncol.20 15.6339

12. Peterson SJ, Mozer M. Differentiating sarcopenia and cachexia among patients with cancer. Nutr Clin Pract. 2017;32(1):30-39. doi: $10.1177 / 0884533616680354$

13. Jeejeebhoy KN. Malnutrition, fatigue, frailty, vulnerability, sarcopenia and cachexia: overlap of clinical features. Curr Opin Clin Nutr Metab Care. 2012;15(3):213-219. doi:10.1097/MCO.0b013e3 $28352694 \mathrm{f}$

14. Lauretani F, Semba RD, Bandinelli S, et al. Carotenoids as protection against disability in older persons. Rejuvenation Res. 2008;11 (3):557-563. doi:10.1089/rej.2007.0581

15. McMillan DC. The systemic inflammation-based Glasgow Prognostic Score: a decade of experience in patients with cancer. Cancer Treat Rev. 2013;39(5):534-540. doi:10.1016/j.ctrv.2012.08.003

16. Fearon K, Strasser F, Anker SD, et al. Definition and classification of cancer cachexia: an international consensus. Lancet Oncol. 2011;12 (5):489-495. doi:10.1016/S1470-2045(10)70218-7

17. Miyake M, Morizawa Y, Hori S, et al. Clinical impact of postoperative loss in psoas major muscle and nutrition index after radical cystectomy for patients with urothelial carcinoma of the bladder. BMC Cancer. 2017;17(1):1-11.

18. Martin L, Birdsell L, MacDonald N, et al. Cancer cachexia in the age of obesity: skeletal muscle depletion is a powerful prognostic factor, independent of body mass index. $J$ Clin Oncol. 2013;31 (12):1539-1547. doi:10.1200/JCO.2012.45.2722

19. Kanda Y. Investigation of the freely available easy-to-use software "EZR" for medical statistics. Bone Marrow Transplant. 2013;48 (3):452-458. doi:10.1038/bmt.2012.244

20. Rier HN, Jager A, Sleijfer S, et al. The prevalence and prognostic value of low muscle mass in cancer patients: a review of the literature. Oncologist. 2016;21(11):1396-1409. doi:10.1634/theoncologist.2016-0066

21. Mangoni AA, Jackson SHD. Age-related changes in pharmacokinetics and pharmacodynamics: basic principles and practical applications. Br J Clin Pharmacol. 2004;57(1):6-14. doi:10.1046/ j.1365-2125.2003.02007.x

22. Loong HH, Ma BBY, Leung SF, et al. Prognostic significance of the total dose of cisplatin administered during concurrent chemoradiotherapy in patients with locoregionally advanced nasopharyngeal carcinoma. Radiother Oncol. 2012;104(3):300-304. doi:10.1016/j. radonc.2011.12.022

23. Hamauchi S, Yokota T, Onozawa Y, et al. Safety and efficacy of concurrent carboplatin plus radiotherapy for locally advanced head and neck cancer patients ineligible for treatment with cisplatin. Jpn J Clin Oncol. 2015;45(12):1116-1121.

24. Katakami N, Uchino J, Yokoyama T, et al. Anamorelin (ONO-7643) for the treatment of patients with non-small cell lung cancer and cachexia: results from a randomized, double-blind, placebocontrolled, multicenter study of Japanese patients (ONO-7643-04). Cancer. 2018;124(3):606-616. doi:10.1002/cncr.31128

25. Garg S, Yoo J, Winquist E. Nutritional support for head and neck cancer patients receiving radiotherapy: a systematic review. Support Care Cancer. 2010;18(6):667-677. doi:10.1007/s00520-009-0686-3 
26. Ravasco P, Monteiro-Grillo I, Vidal PM, Camilo ME. Impact of nutrition on outcome: a prospective randomized controlled trial in patients with head and neck cancer undergoing radiotherapy. Head Neck. 2005;27(8):659-668. doi:10.1002/hed.20221

27. Fietkau R, Riepl M, Kettner H, et al. Supportive use of megestrol acetate in patients with head and neck cancer during radio(chemo) therapy. Eur J Cancer. 1997;33(1):75-79. doi:10.1016/S09598049(96)00309-7
28. Chen HC, Leung SW, Wang CJ, et al. Effect of megestrol acetate and prepulsid on nutritional improvement in patients with head and neck cancers undergoing radiotherapy. Radiother Oncol. 1997;43 (1):75-79. doi:10.1016/S0167-8140(97)01921-X

29. Chen LK, Woo J, Assantachai P, et al. Asian Working Group for Sarcopenia: 2019 consensus update on sarcopenia diagnosis and treatment. J Am Med Dir Assoc. 2020;21(3):300-307.e2. doi:10.1016/j.jamda.2019.12.012

\section{Publish your work in this journal}

Cancer Management and Research is an international, peer-reviewed open access journal focusing on cancer research and the optimal use of preventative and integrated treatment interventions to achieve improved outcomes, enhanced survival and quality of life for the cancer patient
The manuscript management system is completely online and includes a very quick and fair peer-review system, which is all easy to use. Visit http://www.dovepress.com/testimonials.php to read real quotes from published authors. 